\title{
High Q RF transversal filters based on an 80-channel integrated soliton crystal microcomb source
}

Xingyuan Xu, ${ }^{1}$ Mengxi Tan, ${ }^{1}$ Jiayang Wu, ${ }^{1}$ Thach G. Nguyen, ${ }^{2}$ Sai T. Chu, ${ }^{3}$ Brent E. Little, ${ }^{4}$ Roberto Morandotti, ${ }^{5,6,7}$ Arnan Mitchell, ${ }^{2}$ and David J. Moss ${ }^{1, *}$

${ }^{1}$ Centre for Micro-Photonics, Swinburne University of Technology, Hawthorn, VIC 3122, Australia; ${ }^{2}$ ARC Centre of Excellence for Ultrahigh-bandwidth Devices for Optical Systems (CUDOS), RMIT University, Melbourne, VIC 3001, Australia; ${ }^{3}$ Department of Physics and Material Science, City University of Hong Kong, Tat Chee Avenue,

Hong Kong, China; ${ }^{4}$ State Key Laboratory of Transient Optics and Photonics, Xi'an Institute of Optics and Precision Mechanics, Chinese Academy of Science, Xi'an, China; ${ }^{5}$ INRS-Énergie, Matériaux et Télécommunications, 1650 Boulevard Lionel-Boulet, Varennes, Québec, J3X 1S2, Canada; ${ }^{6}$ National Research University of Information Technologies, Mechanics and Optics, St. Petersburg, Russia; ${ }^{7}$ Institute of Fundamental and Frontier Sciences, University of Electronic Science and Technology of China, Chengdu 610054, China. *dmoss@swin.edu.au

\begin{abstract}
We demonstrate a high-Q RF transversal filter based on a 49GHz-FSR integrated microcomb source that provides 80 taps across the C-band. By employing an on-chip micro-ring resonator, we generate a broadband Kerr comb with a large number of comb lines and use it as a high-quality multi-wavelength source. A $Q$ factor of 73.7, an out-of-band rejection of $48.9 \mathrm{~dB}$, and a wide tunable range are demonstrated. The experimental results verify the feasibility of our approach as a solution towards implementing highly reconfigurable MPFs with a large tap number and reduced system complexity.

Keywords: Radio frequency photonics, nonlinear optics, integrated optics
\end{abstract}

\section{INTRODUCTION}

Driven by the ever-increasing demands of broader operation bandwidth, higher frequency selectivity, and lower cost, advanced RF filters are widely researched for applications in modern communication systems [1-3]. While electronic approaches are limited by the "electrical bottleneck", photonic RF filters offer greater performance and many other advantages. Transversal photonic RF filters [4-6] are attractive approaches since they are capable of achieving arbitrary RF transfer functions by simply changing the tap weights. Traditional approaches, including those based on discrete laser arrays or optical frequency comb sources, suffer from drawbacks in one form or another, such as the increased cost and size or the need of high-frequency RF sources. In this paper, we demonstrate a micro-comb based photonic RF transversal filter with a record high number of taps consisting of 80 wavelengths over the $\mathrm{C}$-band. This is the highest number so far reported for microcomb-based RF transversal filters, enabled by a $49 \mathrm{GHz}$-free-spectral-range integrated Kerr micro-comb source [7-10]. This resulted in a $Q_{\mathrm{RF}}$ factor for the RF bandpass filter four times higher than previous results. Further, by programming and shaping the Kerr optical micro-comb, we achieve RF filters with a high out-ofband rejection of up to $48.9 \mathrm{~dB}$ and a wide tunable range. Our experimental results agree well with theory, verifying the feasibility of our approach towards the realization of high performance advanced adaptive RF transversal filters with potentially reduced cost, footprint, and complexity for a wide range of microwave functions [11-27].

\section{EXPERIMENTAL DEMONSTRATIONS}

Figure 1(a) shows a schematic diagram of the 80-tap transversal filter based on micro-combs. To generate Kerr microcombs, the pump power was set at $\sim 30.5 \mathrm{dBm}$ and the wavelength was swept from blue to red. As the detuning was changed further, distinctive soliton crystal optical spectra were generated. The soliton crystal comb was then spectrally shaped via a two-stage optical spectral shaper (Finisar, Waveshaper 4000S). The shaped comb lines were then fed into a dual input optical Mach-Zehnder modulator (MZM) biased at quadrature to yield both positive and negative taps, and then through $\sim 5-\mathrm{km}$ of standard single mode fibre (SMF) to provide the progressive tap delays.

To demonstrate the enhancement in the $Q_{\mathrm{RF}}$ factor, we shaped the micro-comb to implement a low-pass sinc filter featuring equal tap weights for different numbers of taps ranging from 2 to 80 and measured the 3 -dB bandwidth ( $B W_{\text {sinc }}$ ) to calculate the corresponding $Q_{\mathrm{RF}}$ factor $\left(Q_{\mathrm{RF}}=F S R_{\mathrm{RF}} / B W_{\text {sinc }}\right)$. As shown in Fig. 1(b), the measured $B W_{\text {sinc }}$ decreased from 3.962 to $0.236 \mathrm{GHz}$ when the tap number was increased from 2 to 80 , indicating a greatly enhanced $Q_{\mathrm{RF}}$ of up to 
73.7 with 80 taps. To improve the performance of the transversal filter in terms of out-of-band rejection, Gaussian apodization was applied to the sinc filter (Fig. 1(c)). As a function of decreasing root mean square width of the Gaussian function $\sigma$, the main-to-secondary sidelobe ratio of the sinc filter increased from 26.4 to $48.9 \mathrm{~dB}$. In order to demonstrate the tunability of the 80-tap transversal filter's centre frequency, the tap coefficients of the Gaussian-apodized sinc filter were multiplied by a sine function to shift the RF transmission spectrum. The corresponding RF transmission spectra in Fig. 1 (d) shows a tunable centre frequency ranging from $0.05 \times F S R_{\mathrm{RF}}=1.4 \mathrm{GHz}$ to $0.40 \times F S R_{\mathrm{RF}}=11.5 \mathrm{GHz}$ with a relatively high MSSR of $>25 \mathrm{~dB}$.

In conclusion, we demonstrate a microcomb-based photonic RF transversal filters with 80 taps. The experimental results agree well with theory, verifying that our transversal filter is a competitive approach towards achieving advanced adaptive RF transversal filters.
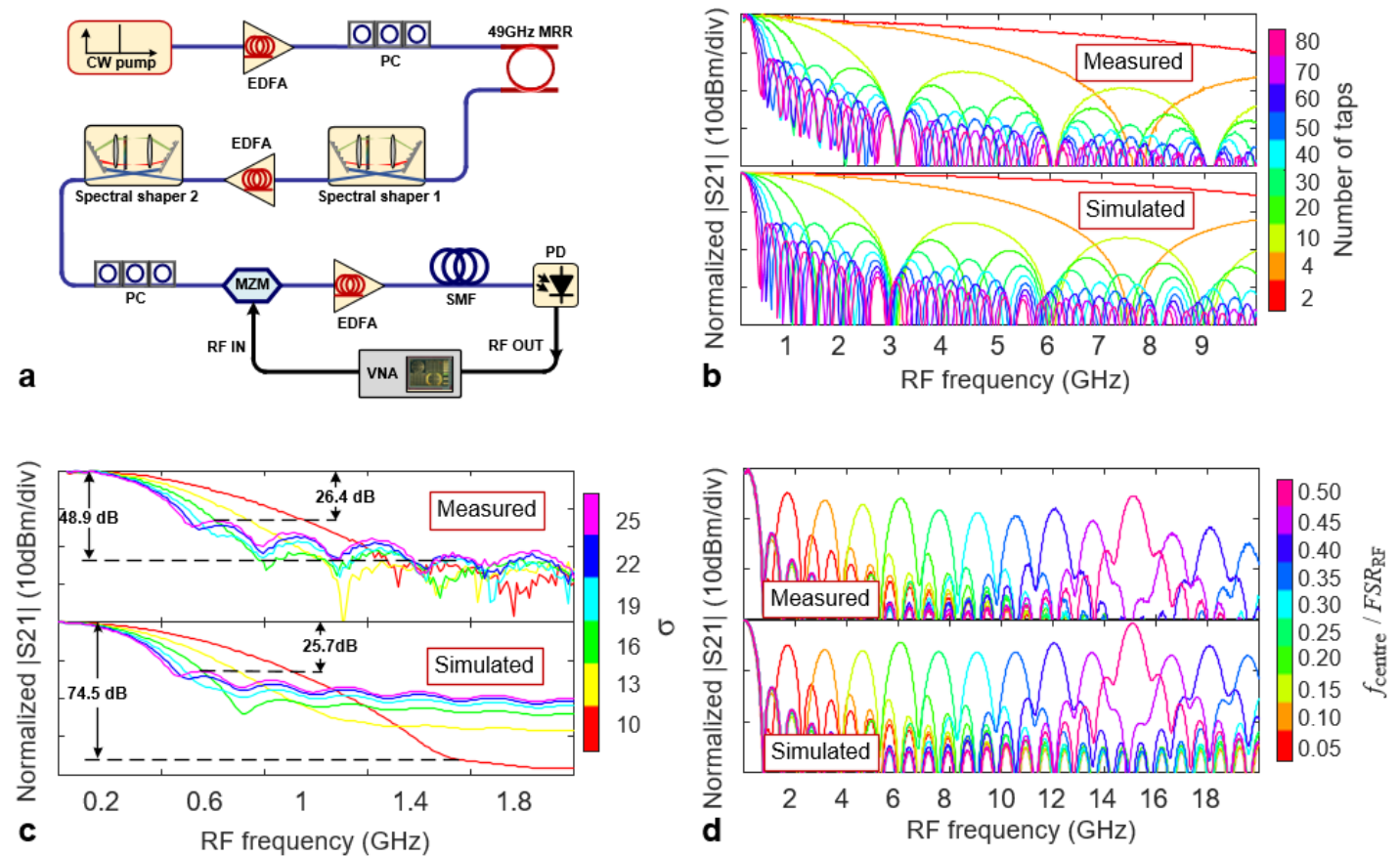

Fig. 1. (a) schematic diagram of the 80-tap photonic RF transversal filter. EDFA: erbium-doped fiber amplifier. PC: polarization controller. PD: photodetector. VNA: vector network analyzer. RF transmission spectra of (b) the sinc filter with different tap numbers, (c) the Gaussian-apodized sinc filter, and (d) tunable bandpass filter.

\section{REFERENCES}

[1] J. Capmany et.al., "Microwave photonics combines two worlds," Nat. Photon. 1, 319-330 (2007).

[2] J. Yao, "Microwave Photonics," J. Lightw. Technol. 27(3), 314-335 (2009).

[3] X. Xie et.al., "Broadband Photonic RF Channelization Based on Coherent Optical Frequency Combs and I/Q Demodulators," IEEE Photon. J. 4(4), 1196-1202 (2012).

[4] D.J. Moss et.al., "New CMOS-compatible platforms based on silicon nitride and Hydex for nonlinear optics," Nat. Photon. 7(8), 597-607 (2013).

[5] X. Xu et.al., "Reconfigurable broadband microwave photonic intensity differentiator based on an integrated optical frequency comb source," APL Photon. 2(9), 096104 (2017).

[6] X. Xu et.al., "Advanced RF and microwave functions based on an integrated optical frequency comb source," Opt. Exp. 26(3), 2569-2583 (2018).

[7] X. Xu et.al., "Photonic microwave true time delays for phased array antennas using a $49 \mathrm{GHz}$ FSR integrated optical micro-comb source," Photon. Res. 6(5), B30-B36 (2018).

[8] X. Xu et.al., "Broadband RF Channelizer based on an Integrated Optical Frequency Kerr Comb Source," J. Lightw. Technol. 36(19), 4519 - 4526 (2018).

[9] X. Xu et.al., "Continuously tunable orthogonally polarized RF optical single sideband generator based on micro-ring resonators," J. Opt. 20(11), 115710 (2018).

[10] J. Wu et.al., "RF Photonics: An Optical Microcombs' Perspective," J. Sel. Top. Quantum Electron. 24(4), 6101020 (2018). 
[11] X. Xu et al., "Orthogonally polarized RF optical single sideband generation and dual-channel equalization based on an integrated microring resonator," Journal of Lightwave Technology, vol. 36, no. 20, pp. 4808-4818. 2018.

[12] T. G. Nguyen et al., "Integrated frequency comb source-based Hilbert transformer for wideband microwave photonic phase analysis," Opt. Express, vol. 23, no. 17, pp. 22087-22097, Aug. 2015.

[13] M. Tan et al., "Broadband microwave and RF photonic fractional Hilbert transformer based on a 50GHz integrated Kerr microcomb", Journal of Lightwave Technology Vol. 37 No. 24, 6097 - 6104, 2019. DOI:10.1109/JLT.2019.2946606

[14] M. Tan et al, "Photonic RF and microwave filters based on 49GHz and 200GHz Kerr microcombs", Optics Communications, vol. 465, Article: 125563. 2020. DOI:10.1016/j.optcom.2020.125563.

[15] X. Xu et al., "Broadband microwave frequency conversion based on an integrated optical micro-comb source", IEEE Journal of Lightwave Technology Vol. 38 No. 2 332-338, 2020. DOI: 10.1109/JLT.2019.2930466.

[16] X. Xu et al., "Microcomb-based photonic RF signal processing", IEEE Photonics Technology Letters Vol. 31 No. 23 1854-1857, 2019. DOI: 10.1109/LPT.2019.2940497.

[17] X. Xu et al., "Advanced adaptive photonic RF filters with 80 taps based on an integrated optical micro-comb source," Journal of Lightwave Technology, vol. 37, no. 4, pp. 1288-1295, 2019. DOI: 10.1109/JLT.2019.2892158.

[18] H. Bao et al., "Laser cavity-soliton microcombs," Nature Photonics, vol. 13, no. 6, pp. 384-389, Jun. (2019).

[19] X. Xu et al., "High performance RF filters via bandwidth scaling with Kerr micro-combs," APL Photonics, vol. 4, no. 2, pp. 026102. 2019.

[20] M. Tan et al., "Broadband microwave and RF fractional differentiator using photonics", IEEE Transactions on Circuits and Systems: Express Briefs, Early Access 37 (2020). DOI:10.1109/TCSII.2020.2965158

[21] X. Xu et al., "Broadband microwave frequency conversion based on an integrated optical micro-comb source", Journal of Lightwave Technology 38 (2) 332-338 (2020). DOI: 10.1109/JLT.2019.2930466

[22] B. Corcoran et al., "Ultra-dense optical data transmission over standard fiber with a single chip source", Nature Communications 11 2568, May 22 (2020). DOI:10.1038/s41467-020-16265-x.

[23] M. Tan et al., "Photonic RF arbitrary waveform generator based on a soliton crystal micro-comb source", Journal of Lightwave Technology Early Access 38 (2020). DOI:10.1109/JLT.2020.3009655.

[24] X. Xu et al., "Broadband photonic radio frequency channelizer with 90 channels based on a soliton crystal microcomb", Journal of Lightwave Technology vol. 38, no. 2, pp. 332-338, 15 Jan.15, (2020). DOI: 10.1109/JLT.2019.2930466.

[25] X.Xu et al., "Photonic RF and microwave integrator with soliton crystal microcombs", IEEE Transactions on Circuits and Systems: Express Briefs, Early Access May 19 (2020). DOI:10.1109/TCSII.2020.2995682

[26] X. Xu et al., "Photonic perceptron based on a Kerr microcomb for scalable high speed optical neural networks", Laser and Photonics Reviews Vol.14, No. 8, 2000070 (2020). DOI:10.1002/lpor.202000070.

[27] X. Xu et al., "Photonic RF phase-encoded signal generation with a microcomb source", Journal of Lightwave Technology, vol. 38, no. 7, pp. 1722-1727 (2020). DOI:10.1109/JLT.2019.2958564. 